\title{
Obtaining the Sky-Temperature Dependence on Voltage Value by the Passive Millimeter Wave Imaging System
}

\begin{abstract}
M. Duman* And A.O. Salman
The passive millimeter wave imaging system contains some of the critical electronic circuits which are antenna, low noise amplifier, detector, and low pass filter. This imaging system is connected to the scanning system that can scan between $15.1^{\circ}$ and $104.9^{\circ}$ in the elevation axis and between $-59.9^{\circ}$ and $59.9^{\circ}$ in the azimuth axis. As a result, the sky temperature can be obtained thanks to elevation axis. The temperature values depend on voltage values because of the system architecture. In this work, the passive millimeter wave imaging system gets the voltage values between $15.1^{\circ}$ and $104.9^{\circ}$ elevation angles but the analysis is made up to $90^{\circ}$ because sky finishes at $90^{\circ}$. There are some obstacles as buildings $70 \mathrm{~m}$ away from the scanning system but the distance is enough to measure the sky temperature. After getting the values from the system, the MatLab Computer Program is run according to these values; consequently, voltage-elevation angle graph or temperature $(\mathrm{K}) \times \alpha$-elevation angle graph is figured out. The experiment were done successfully at 11 a.m. on December 2, 2016 in Umuttepe, İzmit, Kocaeli, Turkey and the weather temperature was $8.7^{\circ} \mathrm{C}$ (or $305.4 \mathrm{~K}$ ), and it was also very cloudy.
\end{abstract}

DOI: 10.12693/APhysPolA.134.346

PACS/topics: imaging system, passive millimeter wave, sky temperature

\section{Introduction of the passive millimeter wave imaging system}

The RADAR (RAdio Detection And Ranging) or SONAR (SOund Navigation And Ranging) systems send the signals and they work with the reflected signals from the objects. As a result, they can help us to understand what it is in front of us [1]. Otherwise, the passive millimeter wave imaging system (PMMWIS) does not send any signal, it only gets the signals from target. This special feature provides economic advantages because sending signals consumes more energy than receiving signals. The cost of production of PMMWIS is also lower than the other active detection systems. Working in bad weather conditions like foggy air is also an important advantage of PMMWIS [2]. There are three important sections in PMMWIS which are scanning system, receiver and processing unit. The sections are explained briefly below:

- The scanning system consists of tripod, dish antenna, receiver holding part and two axis motor. The dish antenna is able to scan in azimuth and elevation axis thanks to the rotating two axis motor. The antenna sends signals to the receiver that is on holding part. The system is on tripod.

- The receiver has five significant parts which are antenna, low noise amplifier (LNA), detector, video amplifier (VA) and low pass filter (LPF).

- Processing unit is the last part of the PMMWIS and it connects to the computer. Passive Millimeter Wave Imager Program and MatLab Program help us to see the images in front of the PMMWIS on computer screen.

* corresponding author; e-mail: dumanmehmet54@gmail.com

\subsection{The scanning system}

There is a two axis motor (2aM) over the tripod and a dish antenna with a diameter of $50 \mathrm{~cm}$ is bonded to the motor. The focal length of the antenna is $20 \mathrm{~cm}$. The scanning system can be seen in Fig. 1a, with receiver and processing unit and in Fig. 1d, with 2aM and dish antenna. The antenna can scan between $15.1^{\circ}$ and $104.9^{\circ}$ in the elevation axis and between $-59.9^{\circ}$ and $59.9^{\circ}$ in the azimuth axis. The antenna can get the voltage values as signals from the between $50 \mathrm{~m}$ and $100 \mathrm{~m}$ away. In this work, the PMMWIS gets the voltage values between $15.1^{\circ}$ and $104.9^{\circ}$ elevation angles but the analysis was done up to $90^{\circ}$ because sky finishes at $90^{\circ}$. All signals are sent to the receiver located at the focal point. The receiver gets the signals from the dish antenna with its own antenna.

\subsection{The receiver}

It is the most important section of the PMMWIS. It is in this part that the signals are purified from the noise, strengthened, amplified and become a process able data. The receiver has its own antenna especially horn or the Vivaldi antenna. Figure $1 \mathrm{~b}$ and $\mathrm{c}$ shows the receiver and its horn antenna. It gets the signals from the scanning system's dish antenna and transmits them to the LNA. After this step, the signals pass through the detector, VA and LPF circuits. It is very important that there should be no power loss at this point.

\subsection{The processing unit}

The processing unit, in Fig. 1a, processes the signals from the LPF and sends them to the computer. Image enhancements are made via programs (PMMW Imager, MatLab, Microsoft Office Picture Manager) on computer and the image is finalized. In this study, there are some matrices or vectors to obtain the sky temperature. They are taken from the numerical value form of image. 


\section{Receiving the signals and processing the data}

The system receives the voltages as signals. Because of the heat and the radiation coming from the sky, each object has its own radiation value. Consequently, the temperature values will depend on the voltage. There will be a constant named alpha. Voltage divided by alpha equals the temperature. In Fig. 1b, an image was given during the scan of PMMWIS. The sky has also its own temperature and radiation. In elevation axis, each angle has specific value of temperature. Voltage values can change when scanning from 0 to 90 degrees. Therefore, PMMWIS can determine the temperature of sky in voltage unit.

\subsection{Weather conditions}

Weather conditions are also very important and must be taken into consideration. According to the experiment on December 2, 2016 at 11:00 a.m. the weather temperature was $8.7^{\circ} \mathrm{C}$ (or $305.4 \mathrm{~K}$ ). It was also very cloudy at this time in İzmit, Kocaeli, Turkey. The PMMWIS can work in these conditions [3]:

- It can work very well in drizzle air.

- It can work in foggy air.

- There is some attenuation but it can work in rain.

- If it is heavy rain, there are $10 \mathrm{~dB}$ or more attenuation per kilometer but it can still work.

In Fig. 1 of Ref. [3], the graph of attenuation by frequency axis is given. It can be seen that the attenuation changes according to the various weather conditions like drizzle, foggy, or rainy. The experiments are done in millimeter wave length. So that, the millimeter-related area should be viewed in the graph.
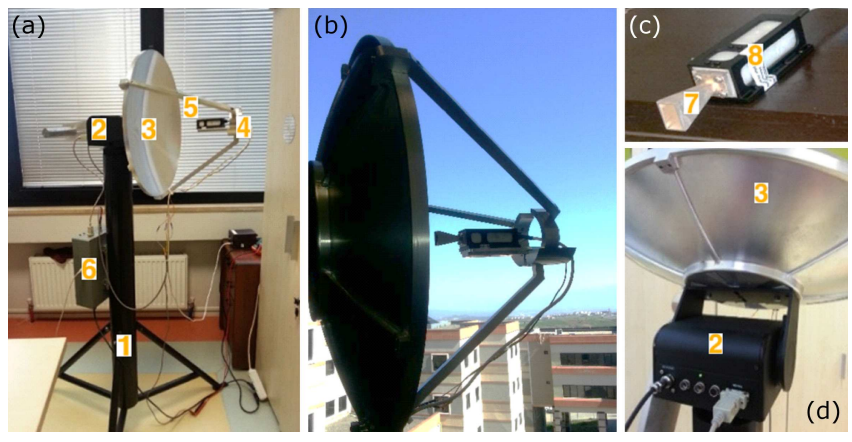

Fig. 1. (a) $1-$ tripod, $2-2 \mathrm{aM}, 3-$ dish antenna, 4 - receiver holding part, 5 - receiver, 6 - processing unit, (b) view from the scanning, (c) 7 - receiver's horn antenna, 8 - receiver's LNA, detector, VA, LPF (inside the metallic box), (d) $2-2 \mathrm{aM}$, 3 - dish antenna.

\subsection{Taking values from the matrix}

PMMWIS performs a full scan and creates a matrix in size of $899 \times 799$. This matrix consists of 899 rows and 799 columns. The first, the last, and another column vector from the middle of the matrix are taken. The graphs are obtained according to these column vectors. In addition, another graph is created with the column vector that is arithmetic mean of the 200th, 300th, 400th, 500th, and 600 th column vectors.

\subsection{The MatLab code}

Here is a short MatLab code to get the arithmetic mean and to combine the column values in the matrix with the elevation scales. The experiment finished on December 2, 2016 at 14:49 p.m. so the file name is 0212161449 .

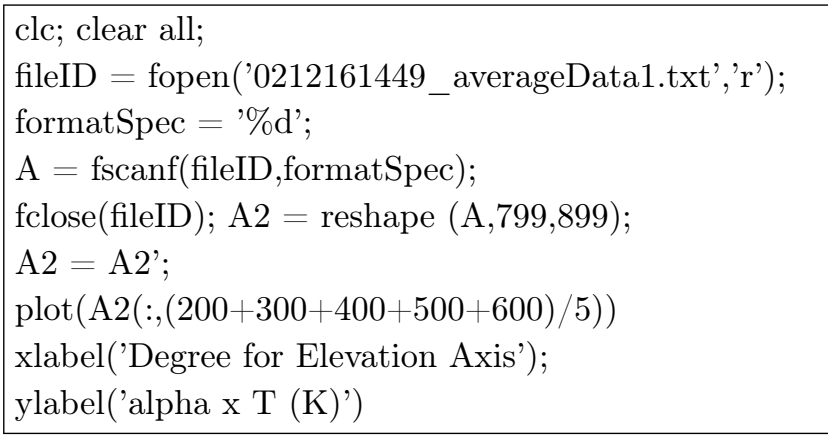

\section{Results and comparison}

The sky finishes at $90^{\circ}$ so the analysis is done up to $90^{\circ}$. In Fig. 2 all elevation grades are given to see that there is no difference between $90^{\circ}$ and $104.9^{\circ}$, because these degrees are not in sky but on ground.

\subsection{Results of the experiment to measure the sky temperature}

The experiment lasted longer than 3 and a half hours. As a result, at about $65^{\circ}$ of elevation axis, there may have been a change of weather. There are some buildings at a distance of about $70 \mathrm{~m}$, so some mistakes may have occurred in the graphic. In example, at $35^{\circ}$ of elevation axis, the vertical axis which is alpha times temperature is about $80 \mathrm{mV}$. It is necessary to find the alpha value in order to find the true temperature value of sky. The alpha value can be got from the other publications for this study.

\subsection{Comparison with other studies}

There is an article about radiometric sky temperature measurements in IEE Proceedings in 1986 from Sayers [4]. The measurements were done in different weather conditions several times. The operating frequencies were 35 and $89 \mathrm{GHz}$. Two of the experiments are similar and they are compared to this study. 


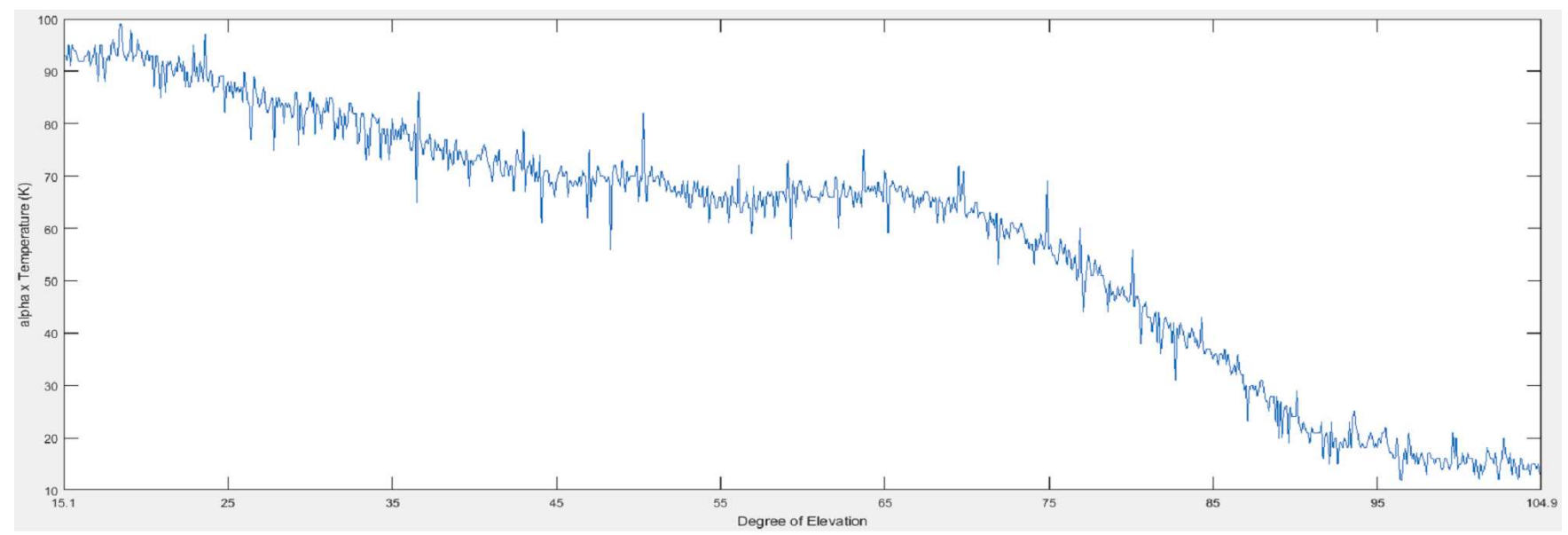

Fig. 2. The graph of sky temperature for this article (alpha $\times$ temperature vs. angle of elevation).

In Figs. 4 and 5 of Ref. [4], these graphs can be seen. The temperature is about $160 \mathrm{~K}$ and $175 \mathrm{~K}$ for $35^{\circ}$ of elevation axis in Ref. [4], Fig. 4 (very large black cloud day, August 13, 1984) and Fig. 5 (overcast day, August $30,1984)$ consecutively. As a result, the alpha value can be found with these equations:

$$
\begin{aligned}
& \text { alpha } \times \text { temperature }[\mathrm{K}]=\text { voltage }[\mathrm{mV}], \\
& \alpha_{2}=80 / 160, \quad \alpha_{2}=0.5\left[\frac{\mathrm{mV}}{\mathrm{K}}\right] \\
& \alpha_{1}=80 / 175, \quad \alpha_{1}=0.457\left[\frac{\mathrm{mV}}{\mathrm{K}}\right]
\end{aligned}
$$

Another article about sky temperature in the microwave region was published in Journal of Meteorology in 1959 from Weger [5]. The experiments were done in different wavelengths and three typical weather conditions. The comparison was done with black body radiation.

\section{Conclusions}

The first, last, and arithmetic vectors are processed and three graphics are created. The closest result to the truth is the graph obtained from the arithmetic mean which can be seen in Fig. 2. The vertical axis can be named voltage or alpha times temperature degrees. The horizontal axis shows elevation grades from 15.1 to 104.9 degrees. In general, it can be said that the experiment is successful and the target is achieved.

\section{Acknowledgments}

This research has been supported by The Scientific and Technological Research Council of Turkey (TÜBİTAK), Kocaeli University and Düzce University.

\section{References}

[1] F.T. Ulaby, R.K. Moore, A.K. Fung, Microwave Remote Sensing. Active and Passive, Vol. 1, Microwave Remote Sensing Fundamentals and Radiometry, Addison-Wesley, 1981.

[2] O.P.N. Calla, Microwave Remote Sensing, in: Defence Res. Dev. Org., DESIDOC, Ministry of Defence, New Delhi 2009.

[3] L. Yujiri, M. Shoucri, P. Moffa, IEEE Microwave Mag. 4, 39 (2003).

[4] A.D. Sayers, IEE Proc. 133, 233 (1986).

[5] E. Weger, J. Meteo. 17, 159 (1960). 